XLVIth Zakopane School of Physics, International Symposium Breaking Frontiers, Zakopane, Poland, May 16-21, 2011

\title{
How Random Is a Random Polymer Coil?
}

\author{
W. ZAJĄC* \\ The Henryk Niewodniczański Institute of Nuclear Physics, Polish Academy of Sciences, Kraków, Poland \\ Short range order within amorphous, random polymer structures is a well known and widely studied property \\ of disordered systems. An aggregate of five random atactic chains, each comprising 400 building units was \\ computer-generated and then used to calculate neutron coherent scattering intensity for $0.01<Q<30 \mathrm{~nm}^{-1}$ \\ $\left(0.001<Q<3 \AA^{-1}\right)$. The latter was compared with experimental data within the diffuse scattering range. On \\ the grounds of quantum chemical calculations for an atactic polystyrene tetramer and the above information it is \\ concluded that interactions among phenyl rings are responsible for the onset of the local order in this polymer.
}

PACS: 61.41.+e, 61.43.-j, 61.05.fg

\section{Introduction}

More than half a century ago Flory showed [1] that a linear polymer chain in a $\theta$ solvent** assumes a Gaussian coil form. An ideal Gaussian coil is modelled by a sequence of identical rigid, zero-volume segments, freely joined to form a random-walk structure. The end-to-end distance in such a structure is then, a random variable, of Gaussian distribution (although restricted to positive arguments), hence the name of a Gaussian coil. Although sometimes useful, this model is unphysical for obvious reasons: in reality not only the same location in space cannot be re-visited in a random walk but joints are not free, either. The first condition is accounted for by developing a self-avoiding random walk approach, and the second one by introducing the concept of an excluded volume. The literature covering various aspects of self-avoiding random walk theory and modelling is now extremely rich (see e.g. [2]).

Properties of polymer chains and their aggregates are typically (although not only) probed with small angle scattering techniques, with a special role of small angle neutron scattering due to specific function of contrast variation through the isotopic substitution.

Finite volume of a polymer building unit and chemical properties of bonds between them are not the only factors influencing the resultant chain conformation. Equally important is a set of physical and chemical interactions that usually lead to the onset of preferred spatial arrangements of atomic groups (chemical sub-units) or, in other words, to the onset of short range order. Such an order can be detected e.g. via diffuse coherent neutron scattering [3]. Diffuse neutron scattering spectra are often difficult to interpret, and the assignment of particular maxima is sometimes a matter of comparative speculation. In

\footnotetext{
* e-mail: wojciech.zajac@ifj.edu.pl

** By definition, the chemical potential of mixing between a polymer and a $\theta$-solvent is zero.
}

recent years, several attempts have been made to reproduce diffuse neutron scattering spectra of random polymer structures by means of calculation based on molecular modelling techniques $[4,5]$. Although very successful, such an approach might not necessarily be unique. In what follows, polystyrene chains are computer-built, arranged in a realistic aggregate, and then the coherent neutron scattering calculated from the first principles.

\section{The model}

A linear polystyrene (PS, see Fig. 1 for the monomer structure) chain was built by head-to-tail joining of 400 monomer units. Dihedral angles defining relative orientation of the $n$-th building unit with respect to the $(n-1)$-th one were allowed to vary randomly within the frameworks of chemical principles. The resultant structure was then subjected to geometry optimisation via the molecular mechanics approach, using the $\mathrm{MM}+$ method (MM2 with additional parameters) [6]. Finally, thermal vibrations were Monte Carlo-simulated for the time long enough to reach equilibrium. This procedure was repeated five times, each time leading to a different random chain of 400 building units. The chains were then arranged in space so that the resultant aggregate had an estimated density of $1 \mathrm{~g} / \mathrm{cm}^{3}$, resembling that of a real bulk polymer $\left(1.05 \mathrm{~g} / \mathrm{cm}^{3}\right)$. Such an aggregate, comprising 2000 PS building units, can already be used for approximate calculations.

Some calculations were also carried out for each of five random chains separately, and then averaged. The purpose of this approach was to compare the results with those of [5], obtained via the amorphous cell method, using relatively short, 100 units polymer chains. In particular, it was interesting to see what features of diffuse coherent neutron scattering spectra can be reproduced assuming no inter-chain correlations. Figure 1 shows the system used for calculations. 


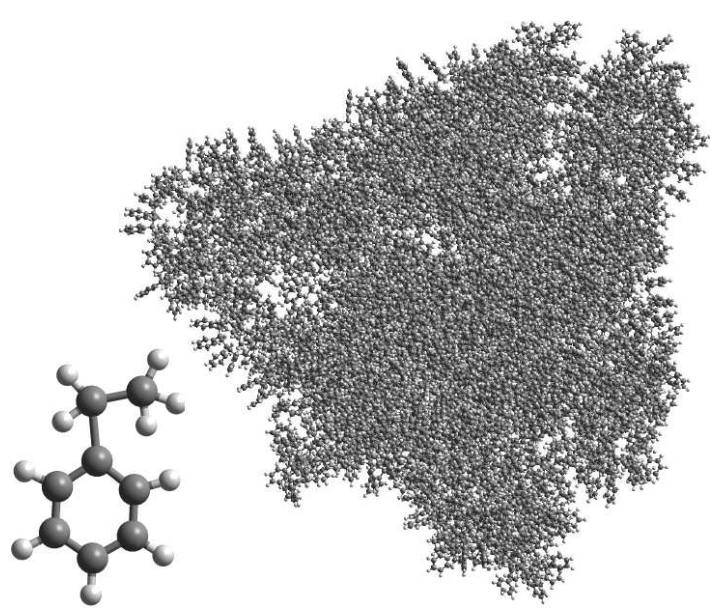

Fig. 1. Polystyrene monomer and the aggregate of five random chains of atactic polystyrene, each comprising 400 building units.

\section{Properties of the simulated PS aggregate}

\subsection{Calculation of fractal dimension}

For the aggregate shown in Fig. 1, the centre of mass was calculated and taken as an origin of an expanding sphere. The mass contained inside such a sphere was calculated and used to estimate the fractal dimension as the slope on the log-log plot, as shown in Fig. 2.

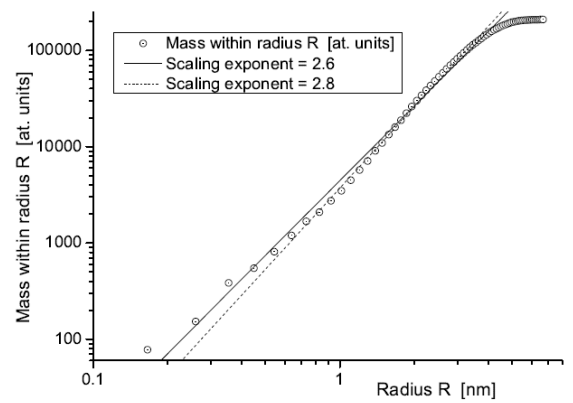

Fig. 2. Total mass of the PS aggregate contained inside a sphere as a function of its radius.

The graph obviously deviates from linear as the sphere radius approaches the surface area of the aggregate. The fractal dimension estimated from a nearly linear part of the graph is somewhere between 2.6 and 2.8, depending on the part of the graph considered. This can be compared with the value of 3 for a homogeneous sphere and the well-known theoretical results of 2 for an ideal Gaussian chain, and $5 / 3$ for a Gaussian coil with excluded volume ("swollen coil").

\subsection{Calculation of coherent neutron scattering}

Given an ensemble of nuclei, the neutron differential cross-section can be expressed by a sum of the coherent and incoherent contributions $[7,8]$ :

$$
\begin{aligned}
& \frac{\mathrm{d} \sigma(\theta)}{\mathrm{d} \Omega}=\langle b\rangle^{2} \sum_{i, j}\left\langle\exp \left(\mathrm{i} \boldsymbol{Q} \boldsymbol{r}_{i j}\right)\right\rangle+\sum_{i}\left(\delta b_{i}\right)^{2} \\
& \equiv\left[\frac{\mathrm{d} \sigma(\theta)}{\mathrm{d} \Omega}\right]_{\mathrm{coh}}+\left[\frac{\mathrm{d} \sigma(\theta)}{\mathrm{d} \Omega}\right]_{\mathrm{inc}},
\end{aligned}
$$

where $\boldsymbol{r}_{i j}=\boldsymbol{r}_{i}-\boldsymbol{r}_{j}$ denotes the relative position of the $j$-th nucleus with respect to nucleus $i, \boldsymbol{Q}$ is the wave vector transfer, and the scattering length of the $i$-th nucleus in the ensemble is expressed in terms of the ensemble-average $\langle b\rangle$ and the varying $\delta b_{i}$ parts

$$
b_{i}=\langle b\rangle+\delta b_{i} .
$$

In studying neutron scattering from polymer chains, most of the theories presented in Veinshtein's book on X-ray diffraction from chain molecules [9] can be utilized, especially in case of partially ordered chains of isotactic or syndiotactic PS, as proposed in [10]. Here, for a random polymer chain, being an ensemble of $N$ nuclei at coordinates $\boldsymbol{r}_{i}$, it is enough to make use of Eq. (1) and to calculate $I_{\mathrm{coh}}(Q)$ assuming various atomic species in the sample

$$
\left[\frac{\mathrm{d} \sigma(\theta)}{\mathrm{d} \Omega}\right]_{\mathrm{coh}}=I_{\mathrm{coh}}(Q)=\frac{1}{N} \sum_{i, j}^{N}\left\langle b_{i} b_{j} \exp \left(\mathrm{i} \boldsymbol{Q} \boldsymbol{r}_{i j}\right)\right\rangle,
$$

where angular brackets indicate spherical averaging over all possible spatial orientations of the polymer coil. It can be shown [11] that Eq. (3) then simplifies to

$$
I_{\text {coh }}(Q)=\frac{1}{N} \sum_{i, j}^{N}\left\langle b_{i}\right\rangle\left\langle b_{j}\right\rangle \frac{\sin Q r_{i j}}{Q r_{i j}} .
$$

Averages $\left\langle b_{i}\right\rangle,\left\langle b_{j}\right\rangle$ include isotopic diversity.

Coherent neutron scattering intensity was calculated according to Eq. (4) within a broad range of $Q$, covering the conventional diffuse scattering region, as well as that of small angle scattering. At this point a statement of disclaim must be made that, any result of such calculation for $Q$ in the small angle region (in practice below $c a$. $5 \mathrm{~nm}^{-1}$ ) has a qualitative meaning only due to a limited and very small size of the "sample", in fact consisting of just one polymer aggregate, and must not be considered a source of information to be compared with any real experiment. Nevertheless, even such imperfect model can be used for a consistency check of the model and the calculations. In particular, overall shape of the log-log plot of $I / I_{0}$ vs. $Q$ in Fig. 3 is correct but the apparent Porod exponent (the slope of the line shown) is at least twice overestimated. The latter seems to be a direct effect of too small the "sample", as test calculations carried out for a smaller ensemble gave the slope still steeper.

Coherent intensity was also calculated for a fully deuterated "sample", as well as for the "samples" partially deuterated (i) at the phenyl rings, and (ii) at the backbone. Figure 3 compares the results of calculations for the fully hydrogenated PS aggregate with the diffuse coherent neutron scattering data measured at the ILL, Grenoble, on a polarised neutron beam with polarisation analysis (for details see e.g. [3]). 

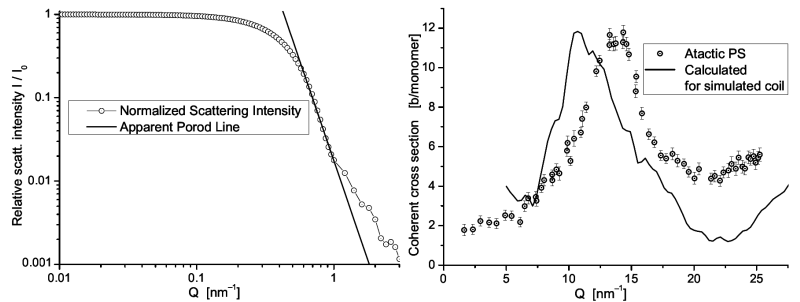

Fig. 3. Calculated coherent neutron scattering intensity for PS aggregate in the small angle and diffuse scattering regions, the latter compared with the experimental neutron diffuse scattering data obtained with polarised neutrons (instrument D7, ILL, Grenoble).

The main feature of the calculated spectrum (above $10 \mathrm{~nm}^{-1}$ ) resembles real data surprisingly well. The only difference can be found in its position on the $Q$-axis. The origin of this shift is not straightforward, as a similar discrepancy is also seen in the coherent intensity computed in [5] by a different method. It can be speculated that a computer-generated PS chain is a more sparse structure than a real PS sample, which could lead to larger correlation distances and, consequently, to coherent scattering maxima at lower $Q$ values. Although the estimated density of the PS aggregate discussed here is that of a real bulk polymer, the microscopic structure of the latter is by no means homogeneous. Small angle scattering experiments on bulk polystyrene reveals a considerable component commonly interpreted as "scattering on the voids" and, consequently, indicates that local density of the polymer may be noticeably higher.

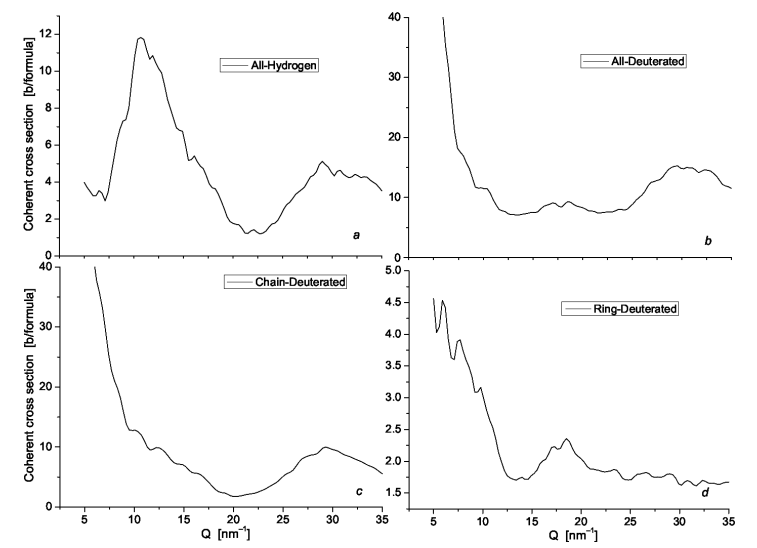

Fig. 4. Calculated coherent neutron scattering intensity for various deuteration types of PS aggregate.

It is tempting to find out the origin of the main peak above $Q=10 \mathrm{~nm}^{-1}$. Figure 4 compares coherent scattering from the PS aggregate under study, calculated for all four combinations of polymer deuteration. Coherent scattering from hydrogenated and deuterated samples is significantly different due to the opposite sign of ${ }^{1} \mathrm{H}$ and ${ }^{2} \mathrm{H}$ coherent scattering lengths $(-3.7406$ and 6.671 , respectively). Here, in the case of a sample composed of hydrogen and carbon atoms only, the negative coherent scattering length of ${ }^{1} \mathrm{H}$ enhances intra-species correlations with respect to inter-species. It does not, however, help to conclude which atomic groups of the PS chain take part in short range correlations resulting in the peak above $Q=10 \mathrm{~nm}^{-1}$. In order to shed some light on this issue a short oligomer of just four PS building units was analysed in more detail.

\subsection{Structure and charge distribution in a PS tetramer}

The chemical structure of an atactic polystyrene tetramer was optimised by means of quantum chemistry calculations, using the DFT method with the B3LYP/6$-311 \mathrm{~g}++(\mathrm{d}, \mathrm{p})$ basis set [12]. The resulting conformation and the computed atomic charges are shown in Fig. 5 and Table.

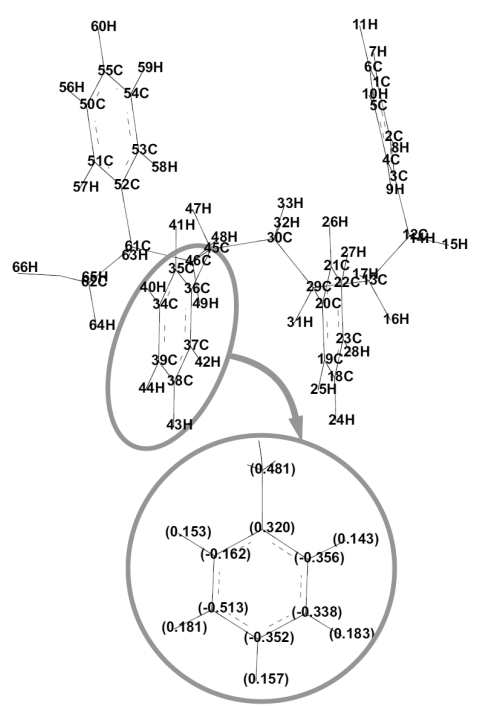

Fig. 5. Optimised conformation of atactic PS tetramer and atomic charges of one phenyl ring (to retain legibility).

As in most chemical structures of this type, carbon atoms in the phenyl rings in positions 2 and 5 (atoms labeled C36 and C39) carry opposite charges, thus creating a local electric dipole at each such ring. The same can be seen at the atomic pairs of $\mathrm{C} 2$ and $\mathrm{C} 6, \mathrm{C} 18$ and C21, C52 and C55. The corresponding entries in Table are highlighted. Most likely there are the electrostatic interactions that make atactic PS assume conformations such that they push neighbouring phenyl rings away at a maximum distance. The same (dipole) electrostatic interactions may then attract distant polymer segments and favour their mutual orientation minimising electrostatic potential energy.

\section{Discussion and conclusions}

There are therefore the ring-ring interactions that are most likely responsible for the local correlations in atactic 
TABLE I PS. This idea is further corroborated by a calculation of

Millikan charges calculated for all atoms in the PS tetramer.

\begin{tabular}{c|c|c|c|c|c}
\hline \hline $\begin{array}{c}\text { At. } \\
\text { label }\end{array}$ & $\begin{array}{c}\text { Millikan } \\
\text { charge }\end{array}$ & $\begin{array}{c}\text { At. } \\
\text { label }\end{array}$ & $\begin{array}{c}\text { Millikan } \\
\text { charge }\end{array}$ & $\begin{array}{c}\text { At. } \\
\text { label }\end{array}$ & $\begin{array}{c}\text { Millikan } \\
\text { charge }\end{array}$ \\
\hline $\mathrm{C} 1$ & -0.346 & $\mathrm{C} 23$ & -0.397 & $\mathrm{C} 45$ & 0.481 \\
$\mathrm{C} 2$ & -0.227 & $\mathrm{H} 24$ & 0.179 & $\mathrm{C} 46$ & -0.144 \\
$\mathrm{C} 3$ & $\underline{0.803}$ & $\mathrm{H} 25$ & 0.153 & $\mathrm{H} 47$ & 0.318 \\
$\mathrm{C} 4$ & -0.172 & $\mathrm{H} 26$ & 0.103 & $\mathrm{H} 48$ & 0.122 \\
$\mathrm{C} 5$ & -0.222 & $\mathrm{H} 27$ & 0.183 & $\mathrm{H} 49$ & 0.131 \\
$\mathrm{C6}$ & $\underline{-0.346}$ & $\mathrm{H} 28$ & 0.158 & $\mathrm{C} 50$ & -0.324 \\
$\mathrm{H} 7$ & 0.177 & $\mathrm{C} 29$ & 0.401 & $\mathrm{C} 51$ & 0.045 \\
$\mathrm{H} 8$ & 0.134 & $\mathrm{C} 30$ & -0.234 & $\underline{\mathrm{C} 52}$ & $\underline{0.309}$ \\
$\mathrm{H} 9$ & 0.143 & $\mathrm{H} 31$ & 0.220 & $\mathrm{C} 53$ & -0.131 \\
$\mathrm{H} 10$ & 0.181 & $\mathrm{H} 32$ & 0.124 & $\mathrm{C} 54$ & -0.342 \\
$\mathrm{H} 11$ & 0.153 & $\mathrm{H} 33$ & 0.070 & $\underline{\mathrm{C} 55}$ & $\underline{-0.372}$ \\
$\mathrm{C} 12$ & -0.883 & $\mathrm{C} 34$ & -0.513 & $\mathrm{H} 56$ & 0.172 \\
$\mathrm{C} 13$ & -0.380 & $\mathrm{C} 35$ & -0.162 & $\mathrm{H} 57$ & 0.161 \\
$\mathrm{H} 14$ & 0.161 & $\underline{\mathrm{C} 36}$ & $\underline{0.320}$ & $\mathrm{H} 58$ & 0.148 \\
$\mathrm{H} 15$ & 0.209 & $\mathrm{C} 37$ & -0.356 & $\mathrm{H} 59$ & 0.179 \\
$\mathrm{H} 16$ & 0.158 & $\mathrm{C} 38$ & -0.338 & $\mathrm{H} 60$ & 0.153 \\
$\mathrm{H} 17$ & 0.144 & $\underline{\mathrm{C} 39}$ & $\underline{-0.352}$ & $\mathrm{C} 61$ & 0.044 \\
$\mathrm{C} 18$ & $\underline{-0.434}$ & $\mathrm{H} 40$ & 0.181 & $\mathrm{C} 62$ & -0.713 \\
$\mathrm{C} 19$ & -0.207 & $\mathrm{H} 41$ & 0.153 & $\mathrm{H} 63$ & 0.226 \\
$\mathrm{C} 20$ & 0.352 & $\mathrm{H} 42$ & 0.143 & $\mathrm{H} 64$ & 0.161 \\
$\mathrm{C} 21$ & $\underline{-0.311}$ & $\mathrm{H} 43$ & 0.183 & $\mathrm{H} 65$ & 0.114 \\
$\mathrm{C} 22$ & -0.353 & $\mathrm{H} 44$ & 0.157 & $\mathrm{H} 66$ & 0.152
\end{tabular}

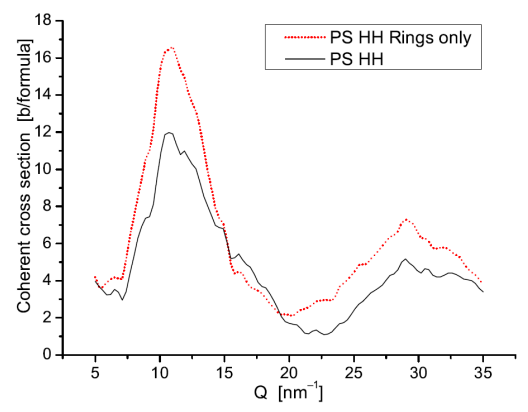

Fig. 6. Calculated coherent neutron scattering from the phenyl rings only of PS aggregate, compared with total coherent scattering. the ring-ring contribution to the coherent scattering of the system in Fig. 1, shown in Fig. 6. The authors of [5] arrive at a similar statement, although reached on the grounds of a different physical system.

A conclusion can therefore be drawn from the results of [5] and from the above example that the central twin peak in the coherent scattering from atactic PS cannot be uniquely attributed to intra- or interchain correlations. Rather, it results from the proximity of the polymer building units and their mutual preferred arrangement, no matter to which chain they belong. This, in turn, indicates that interactions leading to the onset of short-range order may involve small polymer subunits, or chemical structures (groups). This is a clear outcome from the quantum mechanical calculations of the structure and charge distribution of atactic polystyrene tetramer.

\section{References}

[1] P.J. Flory, J. Chem. Phys. 17, 303 (1949).

[2] B.D. Hughes, Random Walks And Random Environments, Vol. 1, Random Walks, Oxford University Press, Oxford 1995.

[3] W. Zajac, Acta Phys. Pol. A 115, 594 (2009).

[4] F. Alvarez, J. Colmenero, R. Zorn, L. Willner, D. Richter, Macromolecules 36, 238 (2003).

[5] I. Iradi, F. Alvarez, J. Colmenero, A. Arbe, Physica B, Condens. Matter 350, E881 (2004).

[6] N.L. Allinger, J. Am. Chem. Soc. 99, 8127 (1977).

[7] G.L. Squires, Introduction to the Theory of Thermal Neutron Scattering, Dover Publications, Mineola 1996.

[8] S.W. Lovesey, The Theory of Neutron Scattering from Condensed Matter, Vol. I, Oxford University Press, Oxford 1986.

[9] B.K. Vainshtein, Diffraction of X-rays Chain Molecules, Elsevier, Amsterdam 1966.

[10] O. Schärpf, on-line report Neutron Scattering by Polymers Using Spin Polarized Neutrons with Polarization Analysis 2008, http://82.135.31.182/polym.pdf .

[11] K. Neumann, O. Schärpf, K. Ziebeck, Physica B, Condens. Matter 180-181, 817 (1992).

[12] J.M. Frisch, W.G. Trucks, B.H. Schlegel, E.G. Scuseria, A.M. Robb, Gaussian 09, Revision A.02.. 